\title{
Distributional properties of Euclidean distances in wireless networks involving road systems
}

\author{
Florian Voss $^{1}$, Catherine Gloaguen ${ }^{2}$, Frank Fleischer ${ }^{3}$, Volker Schmidt ${ }^{1}$
}

\begin{abstract}
Stochastic models for hierarchical telecommunication networks are considered, which can be applied to the analysis and planning of large wireless networks. The network geometry is modelled by random geometric graphs, and the locations of network nodes by point processes on the edges of these graphs. In particular, the locations of high-level components (HLC) are modeled by Cox processes concentrated on the edge sets of random graphs, where their serving zones are the cells of Voronoi tessellations induced by these Cox processes. The locations of low-level components (LLC) are either modeled by planar Poisson processes or by Cox processes concentrated on the same edge sets as the HLC. Distributional properties of distances between the locations of network nodes are closely related with the interference geometry and, consequently, the performance of wireless networks.

Representation formulas are derived for the distribution function and density of the typical Euclidean connection distance between LLC and HLC. They lead to suitable estimators of these characteristics, which can be computed by Monte Carlo simulation of the typical serving zone and the typical segment system in it, respectively.
\end{abstract}

Index Terms : Point processes, Geometric modeling, PoisSON PROCESSES, MONTE CARLO METHOdS, ESTIMATION, INTERFERENCE, MOBILE COMMUNICATION, NETWORKS

\section{INTRODUCTION}

In recent years, wireless networks have been deployed in order to meet the increasing demand for mobile communications. Due to the range of frequencies used and to the increasing number of users, it is necessary to achieve performance and capacity analysis in order to meet the expectation of customers regarding the quality of service (QoS). Estimation of QoS is an important topic for the operator since it reflects the degree of satisfaction that the customers will find using a given service. Thus, specific algorithms have been developed to study how radio resources are shared in the area of an access point (AP), to understand the impact of contention models, for example, or to investigate inter-node connections in sensor networks of mesh WiFi. Such studies are relevant at the scale of the whole network, where the locations of AP and users are assumed to be unknown and modelled following general principles: on regular patterns (grid topology, hexagonal mesh) or randomly as Poisson processes in the plane (homogeneous or non-homogeneous following the users density; [1]). For example, in the development of algorithms in order to derive

e-mail: florian.voss@uni-ulm.de

1. Institute of Stochastics, Ulm University, 89069 Ulm, Germany

2. Orange Labs, 92131 Issy les Moulineaux Cedex 9, France

3. Medical Data Services/Biostatistics, Boehringer-Ingelheim Pharma Gmbh \& Co. KG, 88397 Biberach, Germany contention models for WiFi ([2]), the bandwidth sharing model explicitly needs to express the signal-to-interference ratio as a function of the distance between a user and its AP, both assumed to be located in the plane. In the particular case of WiFi networks deployed in dense urban areas, the typical size of an AP area is in the range of a few hundred meters. Such an area is typically crossed by few street segments. One can then wonder if these streets considered as physical supports for the locations of AP in the performance algorithms could impact on the results.

A first attempt to include street systems into stochastic models for telecommunication networks has been reported e.g. in [3]. Later on, spatial stochastic models for street systems have been considered mainly for the purpose of fixed access network analysis, see e.g. [4], [5], [6]. These random street models depend on a few number of parameters and reproduce the statistical features of the real streets system: number of crossings, length of streets segments, number of quarters, total length of streets measured per unit area. The parameters and choice of best models are recovered by the fitting procedure described in [4]. Hierarchical two-levels networks connecting low-level components (LLC) to their nearest high-level component (HLC) with a physical link that follows the shortest path on the streets, have been developed in this framework ([5]). Since the locations of LLC and HLC as well as the street systems themselves are all modelled by random processes, it is possible, using tools from spatial probability theory, to derive closed analytical formulas for location-dependent quantities. For example, point-to-point length distributions can be given by parametric formulas depending on the parameters of the underlying random street model. We emphasize that this class of models explicitly takes into account the morphology of real street systems; it has been proven to be realistic by comparison of theoretical (parametric) length distributions to connectionlength histograms from real fixed access network data, even in the simplest setting for street models ([6]).

In the context of wireless networks, direct (Euclidean) distances between the locations of network nodes are closely related with the interference geometry and, consequently, the network performance. In particular, in wireless networks with two hierarchy levels one is interested in the direct (Euclidean) distances between the locations of LLC and HLC. Nevertheless, we still assume that LLC and/or HLC are located along street systems, which leads to new classes of non-Poissonian point processes for node locations in wireless networks, see Fig. 1. In this way, we are in a position to offer new parametric formulas for distance distributions that can be used in order to enhance wireless network planning by explicitly taking into 


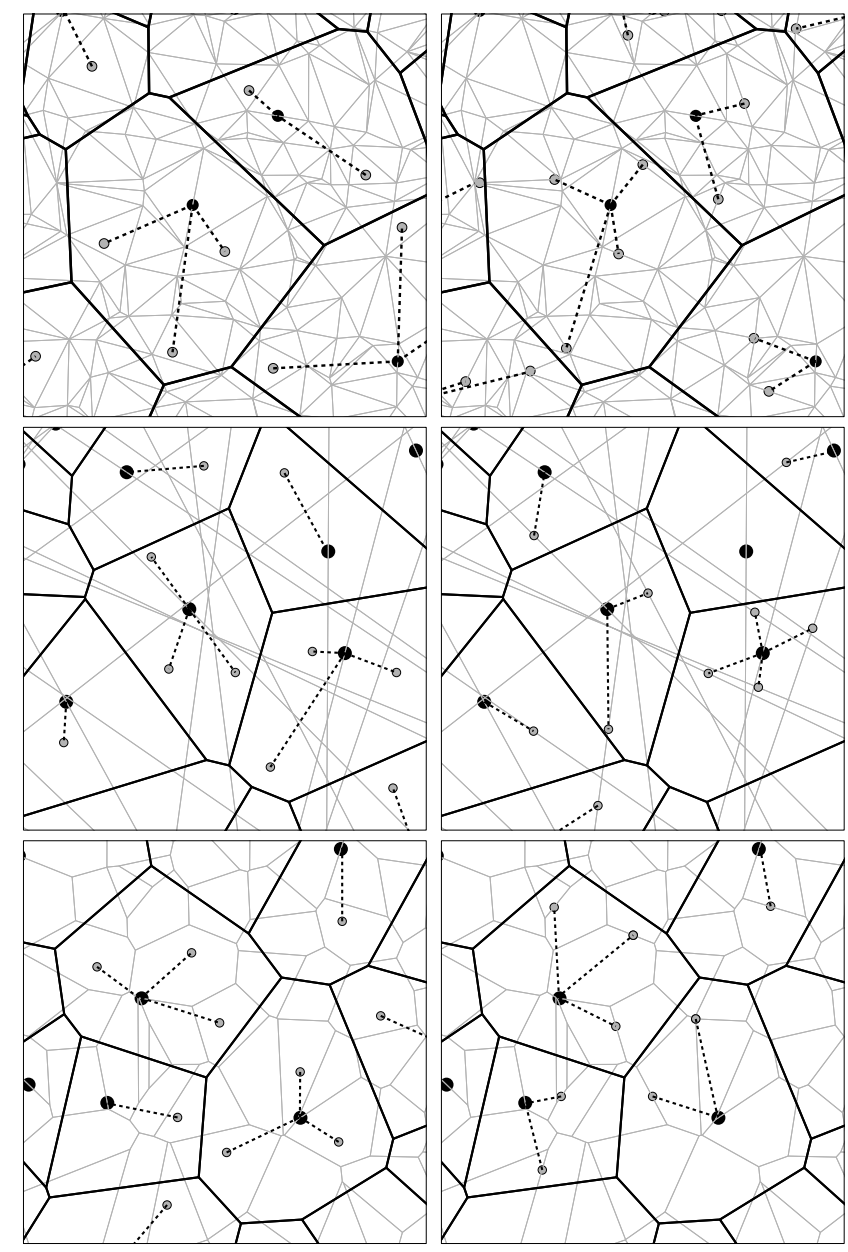

Fig. 1. $\quad H$ on PDT (top), PLT (middle), PVT (bottom) with serving zones (black) and connection distances (dashed) for $L$ Poisson (left) and Cox (right)

account the physical support of network nodes, replacing real street system by random geometric graphs, in particular by the edge sets of random tessellations.

In the present paper, we derive representation formulas for the distribution function and density of typical Euclidean connection distances between LLC and HLC. They lead to suitable estimators of these characteristics, which can be computed by Monte Carlo simulation of the typical serving zone and the typical segment system in it, respectively. Using these simulation algorithms, which we recently developed for the typical cell of several types of random tessellations ([7], [8]), we show that the distributions of Euclidean inter-node distances clearly depend on the model type for the underlying street system, especially for the range of parameters observed at $\mathrm{WiFi}$ scale in dense urban areas. Note that simulation algorithms for the typical serving zone are of independent interest for performance analysis of wireless networks, since these algorithms simultaneously yield a whole configuration of neighboring HLC locations seen from the typical HLC.

The mathematical proofs of all theorems are summarized in the Appendix.

\section{STOCHASTIC MODELING OF SPATIAL NETWORKS}

In this section we describe the kind of telecommunication networks and the stochastic models, which we consider in the present paper. Moreover, we briefly explain some necessary mathematical background and notation. Comprehensive surveys on the usage of stochastic geometry and random geometric graphs in spatial modeling of telecommunication networks can be found in [9], [10]. For further details on marked point processes and random tessellations see e.g. [11], [12], [13], [14] and the Appendix.

\section{A. Stochastic network models with two hierarchy levels}

We consider hierarchical telecommunication networks with two hierarchy levels, i.e., there are LLC and HLC, the location of which are represented by points in the Euclidean plane $\mathbb{R}^{2}$. With each HLC we associate its Voronoi cell as a serving zone and link a LLC to the HLC in whose serving zone it is located. The main goal of our investigation is the analysis of distributional properties of direct connection distances between LLC and HLC, i.e. the Euclidean distances between the locations of LLC and their corresponding HLC. In order to achieve this goal we consider the so-called typical connection distance. It can be interpreted as the Euclidean distance between a location of LLC, which is chosen at random among all locations of LLC, and its nearest HLC.

We assume that HLC are located on the underlying infrastructure of the network, e.g. (inner-city or nationwide) road systems. In our random setting we describe the road system as well as the locations of HLC and LLC by models from stochastic geometry. This reflects the variability of network components and road systems observed in real networks. In a first step we represent the road system by a random geometric graph. We focus on graphs which are given by the edge set of a random tessellation $T=\left\{\Xi_{n}\right\}$, which is a decomposition of $\mathbb{R}^{2}$ into convex and compact polygons $\Xi_{n}$, called the cells of $T$. Note that we can identify $T$ with the random segment system consisting of the segments of the cell boundaries. In the following we will mainly interpret $T$ as a random segment system. For the numerical analysis we consider three cases: $T$ is either a Poisson-Voronoi tessellation (PVT), Poisson-Delaunay tessellation (PDT), or Poisson-Line tessellation (PLT), see Fig. 1. The locations of HLC are modelled by a point process $H=\left\{H_{n}\right\}$, i.e., a locally finite sequence of random points, where we assume that the points of $H$ are located on the segment system $T$. In particular, we assume that $H$ is a Cox point process, whose points are located on $T$. Note that $H$ can be constructed in the following way. For a given realization of $T$ we put linear Poisson processes with (linear) intensity $\lambda_{\ell}>0$ on each segment of $T$, i.e., the number $K$ of points on a segment of length $l$ is $\operatorname{Poi}\left(l \lambda_{\ell}\right)$ distributed and given $K=k$ these $k$ points are independent and uniformly distributed on the segment. Now suppose that $T$ is stationary, i.e., its distribution is invariant with respect to shifts of the origin $o$, then we can define the intensity of $T$ as $\gamma=\mathbb{E} \nu_{1}\left([0,1]^{2} \cap T\right)$, the mean total length of segments per unit area. Then $H$ is stationary as well and its intensity $\lambda=\mathbb{E} \#\left\{n: H_{n} \in[0,1]^{2}\right\}$ is given by $\lambda=\lambda_{\ell} \gamma$. 
The locations of LLC are also modelled by a point process $L=\left\{L_{n}\right\}$, where we distinguish two cases. In the following we either assume that $L$ is a (planar) Poisson process with intensity $\lambda^{\prime}$ or a Cox process on $T$ with (linear) intensity $\lambda_{\ell}^{\prime}$. This reflects either situations where all LLC are located along the infrastructure of the network or LLC are located freely in the plane. A point $L_{n}$ is then linked to its nearest point of $H$, i.e., the serving zones are the cells of the Voronoi tessellation $T_{H}=\left\{\Xi_{H, n}\right\}$ with respect to $H$ and a point $L_{n}$ is linked to the centre of the Voronoi cell in which it is located. The Euclidean distance $D_{n}$ between $L_{n}$ and its nearest point of $H$ is associated to $L_{n}$ as a mark. In this way we can construct the marked point process $L_{D}=\left\{\left[L_{n}, D_{n}\right]\right\}$. Realizations of these modeling components are displayed in Fig. 1.

\section{B. Typical connection distance and typical serving zone}

We are interested in distributional properties of the typical mark $D^{*}$ of $L_{D}$, which can be seen as the typical connection distance from LLC to HLC. Mathematically the typical mark $D^{*}$ is defined by the so-called Palm mark distribution of $L_{D}$. In the ergodic case, the empirical distribution of the marks $D_{n}$ of those points $L_{n}$ belonging to a sampling window $W_{m}$ converges to the distribution of the typical mark $D^{*}$ provided that $W_{m}$ unboundedly increases in all directions, e.g., let $h$ : $\mathbb{R}^{+} \mapsto \mathbb{R}^{+}$and $W_{m}=[-m, m]^{2}$, then

$$
\mathbb{E} h\left(D^{*}\right)=\lim _{m \rightarrow \infty} \frac{1}{\#\left\{n: L_{n} \in W_{m}\right\}} \sum_{L_{n} \in W_{m}} h\left(D_{n}\right) .
$$

Thus, the random variable $D^{*}$ can be interpreted as the Euclidean distance between a location of LLC, which is chosen at random among all points of $L$, and its nearest HLC, i.e., it contains information about spatial averages of the connection distances. Furthermore, $D^{*}$ can be interpreted as the distance from the origin $o$ to the nearest point of $H$ under the condition that there is a point of $L$ located at $o$.

Recall that $T_{H}$ denotes the Voronoi tessellation induced by the Cox process $H$ concentrated on $T$. We use the notation $\Xi_{H}^{*}$ for the typical serving zone of $T_{H}$. Formally, the distribution of $\Xi_{H}^{*}$ can be defined as a Palm mark distribution, where we again have two possible interpretations. On the one hand, the distribution of the typical cell $\Xi_{H}^{*}$ can be seen as the empirical distribution of all cells in a sequence of unboundedly increasing sampling windows, see also equation (II.1), or as the Voronoi cell of $T_{H}$ at $o$ given that $o$ is a point of $H$. Moreover, we use the notation $S_{H}^{*}$ for the segment system of $T$ within the typical cell $\Xi_{H}^{*}$. Then $o \in S_{H}^{*}$ with probability 1 .

\section{Distribution of $D^{*}$ AND ITS STAtisticAL ESTIMATION}

We now derive representation formulas for the distribution function and the probability density of the typical connection distance $D^{*}$ which lead to suitable estimators of these characteristics.

\section{A. Representation by the typical serving zone}

Using Neveu's exchange formula (see Lemma A.1) we can express the distribution function of $D^{*}$ in terms of (i) the typical cell $\Xi_{H}^{*}$ of $T_{H}$ and (ii) the segment system $S_{H}^{*}$ within $\Xi_{H}^{*}$, respectively. This representation does not depend on points of $L$ anymore.

Theorem 3.1: If $L$ is a Poisson process, which is independent of $H$, then the distribution function $F_{D^{*}}:[0, \infty) \rightarrow[0,1]$ of $D^{*}$ is given by

$$
F_{D^{*}}(x)=\lambda_{\ell} \gamma \mathbb{E} \nu_{2}\left(\Xi_{H}^{*} \cap B(o, x)\right), \quad x \geq 0,
$$

where $\nu_{2}\left(\Xi_{H}^{*} \cap B(o, x)\right)$ denotes the area of $\Xi_{H}^{*}$ intersected with the ball $B(o, x) \subset \mathbb{R}^{2}$ centered at $o$ with radius $x$. If $L$ and $H$ are both Cox processes on $T$ which are conditionally independent given $T$, then the distribution function of $D^{*}$ is given by

$$
F_{D^{*}}(x)=\lambda_{\ell} \mathbb{E} \nu_{1}\left(S_{H}^{*} \cap B(o, x)\right), \quad x \geq 0 .
$$

Similar representation formulas can be derived for the probability density of $D^{*}$.

Theorem 3.2: If $L$ is a Poisson process, which is independent of $H$, then the probability density $f_{D^{*}}:[0, \infty) \rightarrow[0, \infty)$ of $D^{*}$ is given by

$$
f_{D^{*}}(x)=\lambda_{\ell} \gamma \mathbb{E} \nu_{1}\left(\Xi_{H}^{*} \cap C(o, x)\right), \quad x \geq 0,
$$

where $\nu_{1}\left(\Xi_{H}^{*} \cap C(o, x)\right)$ denotes the curve length of the circle $C(o, x)$ centred at $o$ with radius $x$ inside $\Xi_{H}^{*}$. If $L$ and $H$ are both Cox processs on $T$ which are conditionally independent given $T$, then the probability density of $D^{*}$ is given by

$$
f_{D^{*}}(x)=\lambda_{\ell} \mathbb{E}\left(\sum_{i=1}^{N_{x}^{*}} \frac{1}{\sin \alpha_{i}^{*}}\right), \quad x \geq 0,
$$

where $N_{x}^{*}=\left|S_{H}^{*} \cap C(o, x)\right|$ is the number of intersection points of the segment system $S_{H}^{*}$ with $C(o, x)$ and $\alpha_{1}^{*}, \ldots, \alpha_{N_{x}^{*}}^{*}$ are the angles at the corresponding intersection points between their tangents to $C(o, x)$ and the intersecting segments.

In the special case that the underlying tessellation $T$ is a PLT, analytical formulas can be derived for the distribution functions $F_{D^{*}}$ considered in (III.2) and (III.3), respectively.

Theorem 3.3: Let $T$ be a PLT. Then, for $x>0$, the distribution function considered in (III.2) is given by

$$
F_{D^{*}}(x)=1-\exp \left(-\lambda_{\ell} \gamma x^{2} \int_{0}^{2} e^{-\lambda_{\ell} x s} \sqrt{4-s^{2}} d s\right),
$$

and the distribution function considered in (III.3) is given by

$F_{D^{*}}(x)=1-e^{-2 \lambda_{\ell} x} \exp \left(-\lambda_{\ell} \gamma x^{2} \int_{0}^{2} e^{-\lambda_{\ell} x s} \sqrt{4-s^{2}} d s\right)$.

Note that analytical formulas can also be derived for the probability densities of $D^{*}$ if $T$ is a PLT, by computing the derivatives of the functions given in (III.6) and (III.7), respectively. However, if the random tessellation $T$ is different from a PLT, then analytical solutions seem to be impossible.

The results of this section can be extended in different ways. For $n \geq 1$, suppose that $\Xi_{H}^{*}$ denotes the $n$-th nearestneighbor Voronoi cell ([12]). Then, for example, $F_{D^{*}}$ given in (III.2) and (III.3) is the distribution function of the typical 
distance from a point of $L$ to its $n$-th nearest neighbor of $H$. Furthermore, if $L$ is a Cox process, it is worth mentioning that $F_{D^{*}}$ coincides with the distribution function of the typical $n$ th nearest-neighbor distance between points of $H$. Node that also these distributions have applications e.g. in interference analysis of wireless networks ([15]).

Finally, we remark that the distribution of $D^{*}$ does not depend on the specific choice of the point process $L$ provided that $H$ is not a Cox process, but a (planar) Poisson process which is independent of $L$. Then, $D^{*}$ has the same distribution as the distance from $o$ to the closest point of $H$, which is Weibull distributed with parameters $\lambda \pi$ and 2, see e.g. [15], [16].

\section{B. Estimators for distribution function and density}

The representation formulas (III.2) - (III.4) easily lead to suitable estimators for the distribution function and probability density of $D^{*}$, which can be computed by Monte-Carlo simulation of $\Xi_{H}^{*}$ and $S_{H}^{*}$. Note that we do not have to simulate $L$. Let $\Xi_{H, 1}^{*}, \ldots, \Xi_{H, n}^{*}$ and $S_{H, 1}^{*}, \ldots, S_{H, n}^{*}$ be $n$ independent copies of $\Xi_{H}^{*}$ and $S_{H}^{*}$, respectively. If $L$ is a Poisson process, then we can define estimators $\widehat{F}_{D^{*}}(x ; n)$ and $\widehat{f}_{D^{*}}(x ; n)$ for the distribution function $F_{D^{*}}(x)$ and the density $f_{D^{*}}(x)$, respectively, by

$$
\widehat{F}_{D^{*}}(x ; n)=\frac{\lambda_{\ell} \gamma}{n} \sum_{i=1}^{n} \nu_{2}\left(\Xi_{H, i}^{*} \cap B(o, x)\right)
$$

and

$$
\widehat{f}_{D^{*}}(x ; n)=\frac{\lambda_{\ell} \gamma}{n} \sum_{i=1}^{n} \nu_{1}\left(\Xi_{H, i}^{*} \cap C(o, x)\right) .
$$

If $L$ is a Cox process, then an estimator $\widehat{F}_{D^{*}}(x ; n)$ for the distribution function $F_{D^{*}}(x)$ is given by

$$
\widehat{F}_{D^{*}}(x ; n)=\frac{\lambda_{\ell}}{n} \sum_{i=1}^{n} \nu_{1}\left(S_{H, i}^{*} \cap B(o, x)\right) .
$$

It is clear that the estimators in (III.8) - (III.10) are unbiased and strongly consistent for fixed $x \geq 0$. However, if $L$ is a Cox process, it is not recommended to use formula (III.5) in order to obtain an analogous estimator $\widehat{f}_{D^{*}}(x ; n)$ for $f_{D^{*}}(x)$ by omitting the expectation in (III.5). Computer experiments showed that this leads to numerical instabilities. In the latter case, it seems to be better first to compute the estimated distribution function $\widehat{F}_{D^{*}}(x ; n)$ by formula (III.10) and then to consider a histogram obtained from this estimated distribution function as an estimator $\widehat{f}_{D^{*}}(x ; n)$ for the density $f_{D^{*}}(x)$. Note that the estimators introduced in this section have many advantages in comparison to estimators based on simulations of the whole model in a large sampling window, which are computationally more intensive and only approximate the distribution of $D^{*}$. Moreover, they demand to simulate also $L$ and thus lead to a larger variability. Furthermore, it is easy to see that even functionals of $D^{*}$, like moments of any order, can be estimated unbiasedly using our estimators for $F_{D^{*}}$ and $f_{D^{*}}$ given in (III.8) - (III.10).

\section{Simulation Algorithms FOR $\Xi_{H}^{*}$ AND $S_{H}^{*}$}

In Section III-B we introduced estimators for the distribution function and density of $D^{*}$ which are based on MonteCarlo simulation of $\Xi_{H}^{*}$ and $S_{H}^{*}$. Thus, we first recall two simulation algorithms for $\Xi_{H}^{*}$ and $S_{H}^{*}$ if $T$ is a PLT or PVT. They have been introduced in [7] and [8], respectively. Furthermore, we propose a new algorithm for the simulation of $\Xi_{H}^{*}$ and $S_{H}^{*}$ if $T$ is a PDT. Such simulation algorithms are of independent interest, since they yield a whole configuration of neighboring HLC locations seen from the typical HLC, which is useful for performance analysis of wireless networks.

\section{A. Cox processes on PLT and PVT}

In [7] a simulation algorithm has been introduced for the typical cell $\Xi_{H}^{*}$ if $T$ is a PLT, which is based on Slivnyak's theorem for stationary Poisson processes. In this case, the typical segment system $S_{H}^{*}$ is directly obtained from the edge set $T$ of the underlying PLT, adding a suitably chosen line through the origin and intersecting with $\Xi_{H}^{*}$.

Furthermore, in [8] direct and indirect simulation algorithms are proposed for the typical cell $\Xi_{H}^{*}$ if $T$ is a PVT. The idea of the indirect algorithm is to simulate cells from which the distribution of $\Xi_{H}^{*}$ can be obtained by a subsequent weighting. Then, the typical segment system $S_{H}^{*}$ can be simulated using a representation formula which is similar to formula (IV.2).

\section{B. Representation formulas for functionals of $\Xi_{H}^{*}$ and $S_{H}^{*}$}

The idea of the indirect simulation algorithm for the typical cell, which has been mentioned in Section IV-A, can be used to simulate the typical cell for Cox processes concentrated on any stationary random tessellation $T$ provided that $T$ can be simulated starting from its typical cell. We show in Section IV-C how a similar idea can be applied in order to get an algorithm for the simulation of random cells from which distributional properties of the typical cell $\Xi_{H}^{*}$, together with its typical segment system $S_{H}^{*}$, can be obtained by a subsequent weighting. In particular, the following general representation formulas are useful.

Let $T$ be an arbitrary stationary tessellation. Then, by $T^{*}$ we denote the Palm version of $T$ with respect to its vertices, i.e., we can assume that $T^{*}$ is distributed according to the conditional distribution of $T$ given that there is a vertex at $o$. Furthermore, let $E^{*}$ denote the union of segments of $T^{*}$ emanating from the origin and let $\mathcal{P}$ denote the family of all convex and compact polygons in $\mathbb{R}^{2}$.

Theorem 4.1: Let $h: \mathcal{P} \rightarrow[0, \infty)$ be a translation-invariant functional. Then,

$$
\mathbb{E} h\left(\Xi_{H}^{*}\right)=\mathbb{E}\left(\nu_{1}\left(E^{*}\right) h(\Xi(Z))\right) / \mathbb{E} \nu_{1}\left(E^{*}\right),
$$

where $\Xi(Z)$ denotes the Voronoi cell with respect to a Cox process $\widetilde{H}$ on $T^{*}$ with linear intensity $\lambda_{\ell}$, around an additional point $Z$. This point $Z$ is conditionally uniformly distributed on $E^{*}$ and conditionally independent of $\widetilde{H}$ given $T^{*}$.

Note that a formula similar to (IV.1) is true for the typical segment system $S_{H}^{*}$. Let $\mathcal{L}$ denote the family of all locally 

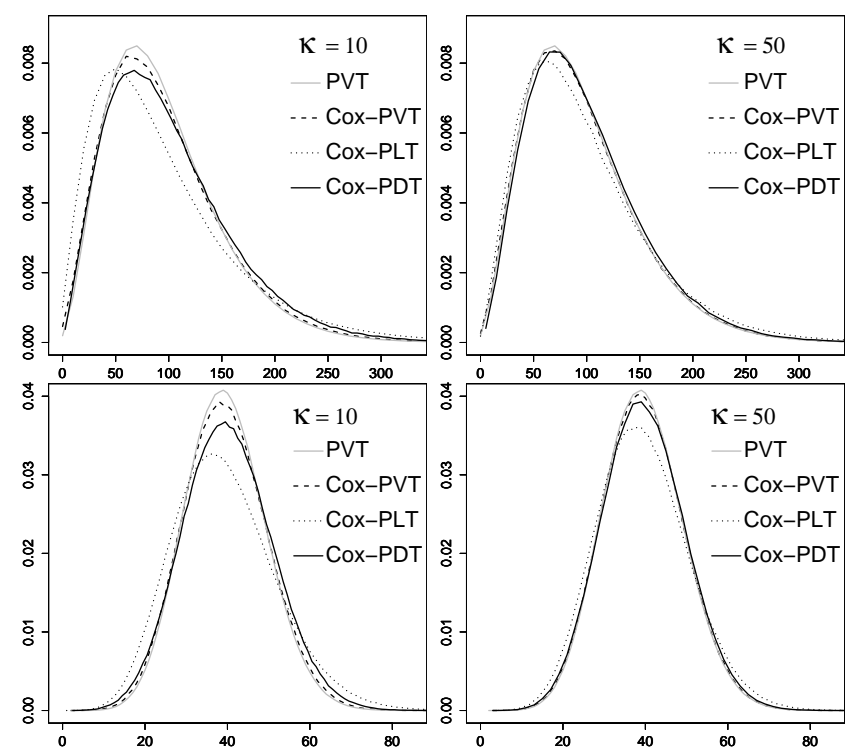

Fig. 2. Histograms for area (top) and perimeter (bottom) of the typical cell

finite segment systems containing the origin. Then, for each translation-invariant functional $h: \mathcal{L} \rightarrow[0, \infty)$, we have

$$
\mathbb{E} h\left(S_{H}^{*}\right)=\mathbb{E}\left(\nu_{1}\left(E^{*}\right) h\left(\Xi(Z) \cap T^{*}\right)\right) / \mathbb{E} \nu_{1}\left(E^{*}\right) .
$$

\section{A new algorithm for Cox processes on PDT}

The formulas (IV.1) and (IV.2) can be easily applied if $T$ is a PDT. Then, the point process $\left\{X_{n}\right\}$ of vertices is Poisson and, using Slivnyak's theorem, the Palm version of $\left\{X_{n}\right\}$ is obtained just by adding a point at the origin. Thus, (IV.1) and (IV.2) lead to the following simulation algorithm for the typical cell $\Xi_{H}^{*}$ and the typical segment system $S_{H}^{*}$.

1) Simulate a planar Poisson process $X=\left\{X_{n}\right\}$ radially with intensity $\mu$, add the origin $o$ to $X$, which gives $X^{*}=X \cup\{o\}$.

2) Construct the union set $E^{*}$ of edges emanating from $o$ of the Delaunay tessellation $T^{*}$ of $X^{*}$.

3) Place $n$ points $\widetilde{H}_{1}, \ldots, \widetilde{H}_{n}$ on $E^{*}$, which are conditionally independent and uniformly distributed on $E^{*}$, given $N=n$, where $N \sim \operatorname{Poi}\left(\lambda_{\ell} \nu_{1}\left(E^{*}\right)\right)$.

4) Place an additional point $Z$ on $E^{*}$, which is independent of $\widetilde{H}_{1}, \ldots, \widetilde{H}_{n}$ and uniformly distributed on $E^{*}$.

5) Construct further edges of $T^{*}$ and place points $\dot{\tilde{H}}_{i}$ of $\widetilde{H}$ on these edges according to linear Poisson processes.

6) Construct the Voronoi cell $\Xi(Z)$ and scale $h(\Xi(Z))$ by $\nu_{1}\left(E^{*}\right) 3 \pi \sqrt{\mu} / 64$.

Note that the weighting factor appears due to the fact that $\mathbb{E} \nu_{1}\left(E^{*}\right)=64 /(3 \pi \sqrt{\mu})$. For further details we refer to [8], where a similar indirect algorithm for the typical cell is given if $T$ is a PVT. If we are interested in distributional properties of $S_{H}^{*}$, then we construct the intersection $\Xi(Z) \cap T^{*}$ and use the same weighting factor $\nu_{1}\left(E^{*}\right) 3 \pi \sqrt{\mu} / 64$.

\section{NUMERICAL RESULTS}

In this section, the underlying tessellation $T$ is assumed to be a PDT, PLT and PVT, respectively.

\section{A. Scaling factor $\kappa$ and Poisson approximation for $\kappa \rightarrow \infty$}

Recall that the Voronoi tessellation $T_{H}$ is fully characterized by two parameters: $\lambda_{\ell}$ and $\gamma$. Moreover, a scaling invariance can be observed which means that structural properties of $T_{H}$ do not change if the $x$-axis and the $y$-axis are scaled in the same way. This implies that with respect to numerical computations it is sufficient to regard only a single parameter $\kappa=\gamma / \lambda_{\ell}$ instead of the originally two-dimensional parameter $\left(\lambda_{\ell}, \gamma\right)$, where the scaling factor $\kappa$ is the mean edge length $\gamma$ of $T$ per unit area divided by the mean number of points $\lambda_{\ell}$ of $H$ per unit length of $T$. If the $x$-axis and the $y$-axis are scaled in the same way, then $\kappa$ remains constant and the same random structure is obtained, but on different scales. Using this invariance property, it suffices to do numerical computations for each $\kappa$ only for one parameter pair $\left(\lambda_{\ell}, \gamma\right)$. For all other parameter pairs with the same scaling factor $\kappa$ these numerical results can then be used to calculate, e.g., estimated mean values of various characteristics by appropriate scaling ([7], [8]).

Furthermore, using tools from the general theory of weak convergence of probability measures ([11], [17]), it can be shown that the Cox process $H$ weakly converges to a stationary Poisson process with intensity $\lambda$ if $\kappa \rightarrow \infty$, where $\gamma \rightarrow \infty$ and $\lambda_{\ell} \rightarrow 0$ such that $\lambda_{\ell} \gamma \rightarrow \lambda$. This is in accordance with the fact that for large $\kappa$ and small $\lambda_{\ell}$ we only get a few points of $H$ per edge of the tessellation $T$ yielding a limit point process without interaction between the points. This implies that the typical connection distance $D^{*}$ as well as further model characteristics like the number of vertices and the perimeter of the typical serving zone $\Xi_{H}^{*}$ converge in distribution to the corresponding characteristics in the Poisson case. Note that this is of practical interest for the numerical results regarding the Cox process $H$ and the Voronoi tessellation $T_{H}$, because we can compare them with corresponding results obtained from analytical formulas known for the Poisson case.

\section{B. Distributional properties of the typical cell}

For different values of $\kappa$ we simulated the typical Voronoi cell $\Xi_{H}^{*}$ if $H$ is a Cox process on $T$ being a PDT, PLT and PVT, respectively, and we estimated the probability densities of such characteristics like the area and perimeter of $\Xi_{H}^{*}$. In [8] some of these characteristics were already investigated for Cox processes on PLT and PVT, respectively, and for the typical cell of PVT. However, in the present paper, we show more comprehensive results including the case of Cox processes on PDT and we discuss the quality of Poisson approximations for these models as $\kappa$ becomes large.

Histograms for the area and perimeter of $\Xi_{H}^{*}$, which are based on 1000000 simulated cells, are displayed in Fig. 2 for two different values of $\kappa$. It can be seen that the estimated probability density of both the area and perimeter of the typical cell of a Cox process on PVT converges very fast to the corresponding density for PVT. One can hardly distinguish between the histograms of Cox processes on PVT and PVT for $\kappa=50$. The rate of convergence for Cox processes on PDT and PLT is not that fast, since for both models there is still 

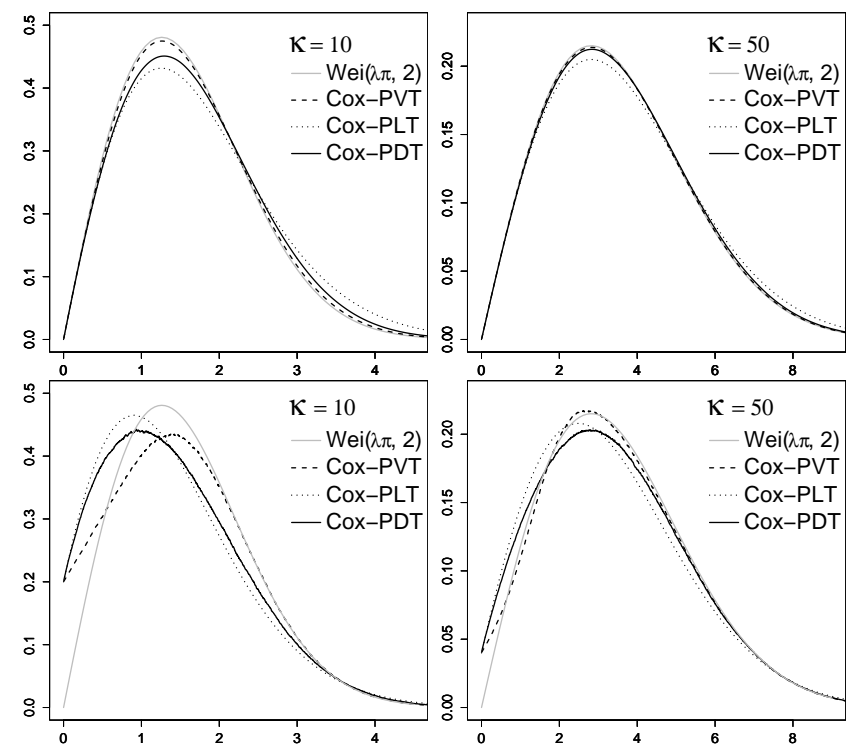

Fig. 3. Density of $D^{*}$ if $L$ is a Poisson process (top) or Cox process (bottom)

a larger difference also for $\kappa=50$, although this difference is less for PDT. This is the behavior one would expect, since the edges of PVT are shorter and more evenly spread in the plane compared to the edges of PDT. Furthermore, the most irregular cells with the longest edges among the three basic models are observed for PLT. So, from this point of view, one expects that the dependencies between the points of $H$ are decreasing faster if $T$ is a PVT rather than for PDT and PLT. This means that the convergence to the PVT is faster for Cox processes on PVT than for PDT which is faster than for PLT.

\section{Probability density of $D^{*}$}

In addition to the scenarios discussed in Section V-B we simulated the typical Voronoi cell $\Xi_{H}^{*}$ together with the typical segment system $S_{H}^{*}$ for Cox processes on $T$ being a PDT and PVT and for different values of $\kappa$, where we put $\gamma=1$. From these simulated data we then estimated the density of $D^{*}$ for $L$ being a Cox process and a Poisson process, respectively, using the estimators introduced in Section III-B. Furthermore, for Cox processes on PLT, the density of $D^{*}$ has been determined by means of (III.6) and (III.7), respectively. The results are displayed in Fig. 3. On the one hand, if $L$ is a Poisson process and $T$ is a PVT, the histogram of $D^{*}$ can hardly be distinguished from that of a $\operatorname{Wei}(\lambda \pi, 2)$-distribution, even for small values of $\kappa$, where the Weibull distribution corresponds to the case that $H$ is a planar Poisson process, see also Section III-A.

If $T$ is a PDT and, especially, if $T$ is a PLT this is not the case. On the other hand, if $L$ is a Cox process, the distribution of $D^{*}$ considerably differs from the $\operatorname{Wei}(\lambda \pi, 2)$-distribution, also in the case that $T$ is a PVT. Then, even for large values of $\kappa$, a clear difference between the Weibull distribution and the distribution of $D^{*}$ can be observed. This discrepancy might be caused by the fact that for a Poisson process $L$ the typical connection distance $D^{*}$ only depends on the shape of the typical cell $\Xi_{H}^{*}$, whereas for a Cox process $L$ it additionally depends on the structure of the typical segment system $S_{H}^{*}$ within $\Xi_{H}^{*}$.

\section{COnclusions And PeRspectives}

We derived estimators for probability densities of internode distances in wireless networks that can be used in order to enhance analysis and planning of wireless networks by explicitly taking into account the physical support of network nodes, replacing real street systems by random geometric graphs. In particular, we showed how the density of the typical connection distance can be efficiently estimated, based on simulations of the typical cell and its typical segment system. This density has been estimated for several models of the underlying street system. It turned out that at the $\mathrm{WiFi}$ scale $(\kappa \approx 10$, see Fig. 3 , bottom left) the density of the typical connection distance clearly depends on the specific choice of the street model and significantly differs from the corresponding density for the case, where the locations of network nodes are assumed to form a (planar) Poisson process.

We introduced a new simulation algorithm for the typical Voronoi cell of Cox processes on PDT. Such simulation algorithms are of independent interest. One reason for this is the necessity to simulate neighboring nodes that shape the typical cell. Thus, we can also recover the joint distance distribution between the center of the typical cell and its first, second, ... closest nodes. This provides an additional approach to performance analysis of meshed WiFi, e.g. in order to investigate if, at constant QoS, it is better to locate nodes on regular patterns (grid topology, hexagonal mesh), or randomly as Poisson processes in the plane, or as Cox processes involving street systems.

Note that the distance densities computed in this paper can be approximated by parametric densities depending on the type of the underlying street model and its parameters, exploiting similar fitting techniques as successfully used in [6] for fixed access networks. We emphasize that even for the simple street models considered in the present paper, the empirical distance densities, which have been computed in [6] from real network data, could be approximated very well by parametric densities. We expect even better results using more sophisticated street models like iterated tessellations ([18]), which is a subject of ongoing research.

We also remark that the method developed in the present paper can be adapted to stochastic models of nationwide telecommunication networks provided that the typical serving zone can be simulated. Possible models for such scenarios include, for example, modulated Poisson-Voronoi tessellations ([19], [20], [21]).

\section{ACKNOWLEDGEMENT}

This research has been supported by Orange Labs (Research agreement 46143714).

\section{REFERENCES}

[1] B. Kauffmann, F. Baccelli, A. Chaintreau, V. Mhatre, K. Papagiannaki, and C. Diot, "Measurement-based self organization of interfering 802.11 wireless access networks." in Proc. IEEE INFOCOM, 2007, pp. 14511459. 
[2] H. Q. Nguyen, F. Baccelli, and D. Kofman, "A stochastic geometry analysis of dense ieee 802.11 networks," in Proc. IEEE INFOCOM, 2007, pp. 1199-1207.

[3] F. Baccelli, M. Klein, M. Lebourges, S. Zuyev, "Stochastic geometry and architecture of communication networks," Telecommunication Systems, vol. 7, pp. 209-227, 1997.

[4] C. Gloaguen, F. Fleischer, H. Schmidt, and V. Schmidt, "Fitting of stochastic telecommunication network models, via distance measures and Monte-Carlo tests," Telecommunication Systems, vol. 31, pp. 353 377,2006

[5] C. Gloaguen, F. Fleischer, H. Schmidt, and V. Schmidt, "Analysis of shortest paths and subscriber line lengths in telecommunication access networks," Networks and Spatial Economics (to appear), 2009.

[6] C. Gloaguen, F. Voss, and V. Schmidt, "Parametric distance distributions for fi xed access network analysis and planning," Preprint (submitted) 2009.

[7] C. Gloaguen, F. Fleischer, H. Schmidt, and V. Schmidt, "Simulation of typical Cox-Voronoi cells, with a special regard to implementation tests," Mathematical Methods of Operations Research, vol. 62, pp. 357-373, 2005.

[8] F. Fleischer, C. Gloaguen, V. Schmidt, and F. Voss, "Simulation of the typical Poisson-Voronoi-Cox-Voronoi cell," Journal of Statistical Computation and Simulation (to appear), 2009.

[9] M. Haenggi, J. G. Andrews, F. Baccelli, O. Dousse, and M. Franceschetti, "Stochastic geometry and random graphs for the analysis and design of wireless networks," IEEE Journal on Selected Areas in Communications (to appear), 2009.

[10] S. Zuyev, "Stochastic geometry and telecommunications networks," in: Stochastic Geometry: Highlights, Interactions and New Perspectives, W.S. Kendall and I. Molchanov, Eds., Oxford University Press (to appear), 2009.

[11] D. Daley and D. Vere-Jones, An Introduction to the Theory of Point Processes. New York: Springer, 2003/08, vol. I/II.

[12] A. Okabe, B. Boots, K. Sugihara, and S. N. Chiu, Spatial Tessellations, 2nd ed. Chichester: J. Wiley \& Sons, 2000.

[13] R. Schneider and W. Weil, Stochastic and Integral Geometry. Berlin: Springer, 2008.

[14] D. Stoyan, W. S. Kendall, and J. Mecke, Stochastic Geometry and its Applications, 2nd ed. Chichester: J. Wiley \& Sons, 1995.

[15] M. Haenggi, "On Distances in Uniformly Random Networks," IEEE Trans. on Information Theory, vol. 51, no. 10, pp. 3584-3586, 2005.

[16] S. Srinivasa and M. Haenggi, "Distance distributions in fi nite uniformly random networks: Theory and applications." Preprint, 2008.

[17] P. Billingsley, Convergence of Probability Measures, 2nd ed. New York: J. Wiley \& Sons, 1999.

[18] R. Maier, J. Mayer, and V. Schmidt, "Distributional properties of the typical cell of stationary iterated tessellations," Mathematical Methods of Operations Research, vol. 59, pp. 287-302, 2004.

[19] B. Błaszczyszyn and R. Schott, "Approximate decomposition of some modulated Poisson-Voronoi tessellations," Advances in Applied Probability, vol. 35, pp. 847-862, 2003.

[20] B. Błaszczyszyn and R. Schott, "Approximations of functionals of some modulated Poisson-Voronoi tessellations with applications to modeling of communication networks," Japan J. Indust. Appl. Math., vol. 22, pp. 179-204, 2005.

[21] F. Fleischer, C. Gloaguen, H. Schmidt, V. Schmidt, and F. Schweiggert, "Simulation algorithm of typical modulated Poisson-Voronoi cells and application to telecommunication network modelling," Japan J. Indust. Appl. Math., vol. 25, pp. 305-330, 2008.

[22] J. Neveu, "Sur les mesures de palm de deux processus ponctuels stationnaires," Zeitschrift für Wahrscheinlichkeitstheorie und verwandte Gebiete, vol. 34, pp. 199-203, 1976.

[23] E. B. V. Jensen, Local Stereology. Singapore: World Scientifi c Publ Co., 1998.

[24] R. E. Miles, "A synopsis of 'Poisson flats in Euclidean spaces'," in Stochastic Geometry, E. F. Harding and D. G. Kendall, Eds. Wiley, 1974, pp. 202-227.

\section{APPENDIX}

\section{A. Marked point processes and Neveu's exchange formula}

A marked point process $X=\left\{\left[X_{n}, M_{n}\right]\right\}$ with some (Polish) mark space $(\mathbb{M}, \mathcal{B}(\mathbb{M}))$ can be regarded as a random element of the measurable space $\left(N_{\mathbb{M}}, \mathcal{N}_{\mathbb{M}}\right)$, where $N_{\mathbb{M}}$ denotes the family of all sequences $\left\{\left(x_{n}, m_{n}\right)\right\} \subset \mathbb{R}^{2} \times \mathbb{M}$ such that $\left\{x_{n}\right\} \subset \mathbb{R}^{2}$ is locally finite, $\left\{m_{n}\right\} \subset \mathbb{M}$, and $\mathcal{N}_{\mathbb{M}}$ denotes the usual $\sigma$-Algebra defined on $N_{\mathbb{M}}$. We say that $X$ is stationary if $X$ has the same distribution as $t_{x} X=$ $\left\{\left[X_{n}-x, M, n\right]\right\}$ for all $x \in \mathbb{R}^{2}$. Then the intensity of $X$ is given by $\lambda=\mathbb{E} \#\left\{X_{n} \in[0,1]^{2}\right\}$. In the following proofs we frequently utilize Neveu's exchange formula ([22]) for two jointly stationary marked point processes $X^{(1)}=\left\{\left[X_{n}^{(1)}, M_{n}^{(1)}\right]\right\}$ and $X^{(2)}=\left\{\left[X_{n}^{(2)}, M_{n}^{(2)}\right]\right\}$ with marks in $\mathbb{M}_{1}$ and $\mathbb{M}_{2}$, respectively. Define $Y=\left(X^{(1)}, X^{(2)}\right), N_{\mathbb{M}_{1}, \mathbb{M}_{2}}=N_{\mathbb{M}_{1}} \times N_{\mathbb{M}_{2}}$ and let $\lambda^{(1)}$ and $\lambda^{(2)}$ denote the intensities of $X^{(1)}$ and $X^{(2)}$, respectively. Then the Palm distributions $\mathbb{P}_{Y}^{(i)}, i=1,2$ on $\mathcal{N}_{\mathbb{M}_{1}} \otimes \mathcal{N}_{\mathbb{M}_{2}} \otimes \mathcal{B}\left(\mathbb{M}_{i}\right)$ with respect to the $i$-th component of $Y$ are defined by

$\mathbb{P}_{Y}^{(i)}(A \times G)=\frac{\mathbb{E} \#\left\{n: X_{n}^{(i)} \in[0,1)^{2}, M_{n}^{(i)} \in G, t_{X_{n}^{(i)}} Y \in A\right\}}{\lambda^{(i)}}$

for any $A \in \mathcal{N}_{\mathbb{M}_{1}} \otimes \mathcal{N}_{\mathbb{M}_{2}}$ and $G \in \mathcal{B}\left(\mathbb{M}_{i}\right)$. Here $t_{x} Y=$ $\left(t_{x} X^{(1)}, t_{x} X^{(2)}\right)$ is obtained if we shift $X^{(1)}$ and $X^{(2)}$ simultaneously. Note that for any $A \in \mathcal{N}_{\mathbb{M}_{i}}, G \in \mathcal{B}\left(\mathbb{M}_{i}\right)$ we get that $\mathbb{P}_{Y}^{(1)}\left(A \times N_{\mathbb{M}_{2}} \times G\right)=\mathbb{P}_{X^{(1)}}^{*}(A \times G)$ and $\mathbb{P}_{Y}^{(2)}\left(N_{\mathbb{M}_{1}} \times A \times G\right)=\mathbb{P}_{X^{(2)}}^{*}(A \times G)$, where $\mathbb{P}_{X^{(1)}}^{*}$ and $\mathbb{P}_{X^{(2)}}^{*}$ is the ordinary Palm distribution of the marked point process $X^{(1)}$ and $X^{(2)}$, respectively. Using the notation $\psi=$ $\left(\psi^{(1)}, \psi^{(2)}\right)$ for the elements of $N_{\mathbb{M}_{1}, \mathbb{M}_{2}}$, Neveu's exchange formula takes the following form, see e.g. [18].

Lemma A.1: For $f: \mathbb{R}^{2} \times \mathbb{M}_{1} \times \mathbb{M}_{2} \times N_{\mathbb{M}_{1}, \mathbb{M}_{2}} \rightarrow[0, \infty)$, it holds that

$$
\begin{aligned}
& \lambda^{(1)} \int \sum_{\left(x, m_{2}\right) \in \psi^{(2)}} f\left(x, m_{1}, m_{2}, t_{x} \psi\right) \mathbb{P}_{Y}^{(1)}\left(d\left(\psi, m_{1}\right)\right) \\
& =\lambda^{(2)} \int \sum_{\left(x, m_{1}\right) \in \psi^{(1)}} f\left(-x, m_{1}, m_{2}, \psi\right) \mathbb{P}_{Y}^{(2)}\left(d\left(\psi, m_{2}\right)\right) .
\end{aligned}
$$

\section{B. Proof of Theorem 3.1}

We apply Lemma A.1 to $\left(T_{H}, L_{D}\right)$ in order to prove Theorem 3.1. Note that $T_{H}=\left\{\left[H_{n}, \Xi_{H, n}^{o}\right]\right\}$ can be regarded as a marked point process, where $H_{n}$ is marked with its shifted Voronoi cell $\Xi_{H, n}^{o}=\Xi_{H, n}-H_{n}$. Put $\mathbb{M}_{1}=\mathcal{P}, \mathbb{M}_{2}=[0, \infty)$ and, for some measurable $h:[0, \infty) \rightarrow[0, \infty)$, consider the function $f: \mathbb{R}^{2} \times \mathcal{P} \times[0, \infty) \times N_{\mathcal{P},[0, \infty)} \rightarrow[0, \infty)$ given by $f(x, \xi, m, \psi)=h(m)$ if $o \in \xi+x$, and $f(x, \xi, m, \psi)=0$ otherwise. Then, applying Lemma A.1, we get that $\mathbb{E} h\left(D^{*}\right)=$ $\lambda_{\ell} \gamma\left(\lambda^{\prime}\right)^{-1} \mathbb{E}\left(\mathbb{E}\left(\sum_{L_{n} \in \Xi_{H}^{*}} h\left(D_{n}\right) \mid \Xi_{H}^{*}\right)\right)$, where $\lambda^{\prime}$ denotes the intensity of $L$. If $L$ is a Poisson process, we have

$$
\begin{aligned}
\mathbb{E}\left(\sum_{L_{n} \in \Xi_{H}^{*}} h\left(D_{n}\right) \mid \Xi_{H}^{*}\right) & =\sum_{k=1}^{\infty} \frac{\lambda^{k}}{e^{\lambda} k !} \sum_{i=1}^{k} \int_{\Xi_{H}^{*}} \ldots \int_{\Xi_{H}^{*}} h\left(\left|u_{i}\right|\right) \frac{d u_{1} \ldots d u_{k}}{\nu_{2}\left(\Xi_{H}^{*}\right)^{k}} \\
& =\lambda^{\prime} \int_{\Xi_{H}^{*}} h(|u|) \nu_{2}(d u),
\end{aligned}
$$

where $\lambda=\lambda^{\prime} \nu_{2}\left(\Xi_{H}^{*}\right)$. On the other hand, if $L$ is a Cox process on $T$, we have $\lambda^{\prime}=\lambda_{\ell}^{\prime} \gamma$ and similar as above we get

$$
\mathbb{E}\left(\sum_{L_{n} \in \Xi^{*}} h\left(D_{n}\right) \mid \Xi_{H}^{*}\right)=\lambda_{\ell}^{\prime} \int_{S_{H}^{*}} h(|u|) \nu_{1}(d u),
$$

see also [21], [5]. Thus, choosing $h(m)=\mathbb{1}_{[0, x]}(m)$, the proof is completed. 


\section{Proof of Theorem 3.2}

If $L$ is a Poisson process, we can use the polar decomposition of the Lebesgue measure $\nu_{2}$ to get from (III.2) that

$$
\begin{aligned}
F_{D^{*}}(x) & =\lambda_{\ell} \gamma \mathbb{E} \int_{0}^{\infty} \int_{0}^{2 \pi} y \mathbb{I}_{\Xi_{H}^{*} \cap B(o, x)}(y, \phi) d \phi d y \\
& =\lambda_{\ell} \gamma \int_{0}^{x} \mathbb{E} \nu_{1}\left(\Xi_{H}^{*} \cap C(o, y)\right) d y
\end{aligned}
$$

where $z=(y, \phi)$ is the representation of $z \in \mathbb{R}^{2}$ in polar coordinates. If $L$ is a Cox process, we can use the fact that $\nu_{1}\left(S_{H}^{*} \cap B(o, x)\right)=\int_{0}^{x} \sum_{i=1}^{N_{y}^{*}} \sin ^{-1} \alpha_{i}^{*} d y$, where this decomposition of the Hausdorff measure $\nu_{1}$ can be derived using Theorem 2.1 of [23]. Then, in the same way as above, we get that $F_{D^{*}}(x)=\lambda_{\ell} \int_{0}^{x} \mathbb{E}\left(\sum_{i=1}^{N_{y}^{*}} \sin ^{-1} \alpha_{i}^{*}\right) d y$, which proves Theorem 3.2.

\section{Proof of Theorem 3.3}

Let $H$ be a Cox process concentrated on the PLT $T$ and $L$ a Poisson process which is independent of $H$. Then, using the Independence of $L$ and $H$, we get that for each $x \geq 0$ that $F_{D^{*}}(x)=1-\mathbb{P}\left(\#\left\{H_{n} \in B(o, x)\right\}=0\right)$ and

$$
\begin{aligned}
\mathbb{P}\left(\#\left\{H_{n} \in B(o, x)\right\}=0\right) & =\mathbb{E} \exp \left(-\lambda_{\ell} \nu_{1}(B(o, x) \cap T)\right) \\
& =\mathbb{E} \exp \left(-\lambda_{\ell} x \nu_{1}\left(B(o, 1) \cap \frac{1}{x} T\right)\right) .
\end{aligned}
$$

The latter expectation can be determined in the following way. Let $M$ denote the random number of lines of $T$ which intersect $B(o, x)$. Then $M$ has a Poisson distribution with parameter $2 \gamma x$ and, given $M=k$, these $k$ lines are independent and isotropic uniform random (IUR) lines ([24]). Thus for $\mathbb{P}(M=$ $k):=p_{k}$ we get

$$
\begin{aligned}
& \mathbb{E} \exp \left(-\lambda_{\ell} x \nu_{1}\left(B(o, 1) \cap \frac{1}{x} T\right)\right) \\
& =\sum_{k=0}^{\infty} p_{k} \mathbb{E}\left(\exp \left(-\lambda_{\ell} x \nu_{1}\left(B(o, 1) \cap \frac{1}{x} T\right)\right) \mid M=k\right) \\
& =\exp (-2 \gamma x) \sum_{k=0}^{\infty} \frac{\left(2 \gamma x \mathbb{E} \exp \left(-\lambda_{\ell} x I\right)\right)^{k}}{k !} \\
& =\exp \left(-2 \gamma x\left(1-\mathbb{E} \exp \left(-\lambda_{\ell} x I\right)\right)\right),
\end{aligned}
$$

where $I$ is the intersection length of an IUR line hitting $B(o, 1)$. The distribution function of $I$ can be calculated as $F_{I}(s)=1-\sqrt{1-s^{2} / 4}$ for $s \in[0,2]$. By partial integration we get that $\mathbb{E} \exp \left(-\lambda_{\ell} x I\right)=1-$ $\left(\lambda_{\ell} / 2\right) x \int_{0}^{2} e^{-\lambda_{\ell} x s} \sqrt{4-s^{2}} d s$, which proves (III.6). If $L$ is a Cox process, then we can do basically the same calculations. But, due to Slivnyak's theorem, we then have one additional line through the origin with probability 1 which yields the additional factor $\exp \left(-2 \lambda_{\ell} x\right)$ in (III.7).

\section{E. Proof of Theorem 4.1}

We regard the random element $Y=\left(T_{H}, T\right)$ of $N_{\mathcal{P}, \mathcal{L}}$, i.e., we regard $T_{H}$ as $H$ marked with the centered Voronoi cells and $T$ as its vertices marked with the centered segments emanating from them. Let $f: \mathbb{R}^{2} \times \mathcal{P} \times \mathcal{L} \times N_{\mathcal{P}, \mathcal{L}} \rightarrow[0, \infty)$ be given by $f(x, \xi, \zeta, \psi)=h(\xi)$ if $o \in \zeta+x$, and $f(x, \xi, \zeta, \psi)=0$ otherwise. Then we get

$$
\mathbb{E} h\left(\Xi_{H}^{*}\right)=\frac{1}{2} \int \sum_{(x, \zeta) \in \psi^{(2)}} f\left(x, \xi, \zeta, t_{x} \psi\right) \mathbb{P}_{Y}^{(1)}(d(\psi, \xi)) .
$$

Now we can use again Lemma A.1 and the same arguments as in the proof of Theorem 3.5 in [8] in order to get that $\mathbb{E} h\left(\Xi_{H}^{*}\right)=\left(\lambda_{\ell} \mu /(2 \lambda)\right) \mathbb{E}\left(\nu_{1}\left(E^{*}\right)(h(\Xi(Z)))\right.$, where $\mu$ is the intensity of the point process of vertices of $T$. Furthermore, $\lambda=\lambda_{\ell} \mathbb{E} \nu_{1}\left(T \cap[0,1]^{2}\right), \mathbb{E} \nu_{1}\left(E^{*}\right)=(2 / \mu) \mathbb{E} \nu_{1}\left(T \cap[0,1]^{2}\right)$. This completes the proof. 